\title{
Knowledge Sharing Network Based Trust Management for Agent-Mediated E- Commerce
}

\author{
Jing Lei ${ }^{1,2}$, Xixiang Zhang ${ }^{1}$ and Bao'an Yang ${ }^{1}$ \\ 1.Glorious Sun School of Business\& Management, Donghua University, \\ Shanghai 200051, China \\ 2 Shanghai Business School, Shanghai 2000235, China \\ leijing1289@sina.com
}

\begin{abstract}
The efficiency of e-commerce can be increased through the usage of intelligent agents which negotiate and execute contracts on behalf of their owners. The knowledge of trust to secure interactions between autonomous agents is crucial for the success of agent-mediated e-commerce. Building a knowledge sharing network among peer agents helps to overcome trust-related boundaries in an intelligent environment where least human intervention is desired. Based on this network, this paper proposes a trust management model integrating external trustworthiness ratings from other peer agents and the internal assessment of past experiences with the peer node. Knowledge of trust is developed over time through learning from prior business interactions and problems related to peer agents' dishonesty can be solved.
\end{abstract}

\section{Introduction}

With the development and popularity of e-commerce, intelligent agents are being employed to automate time and resource consuming tasks such as service discovery, service selection, contract negotiation, business execution and quality of service reviews. The efficiency of e-commerce can be increased through the usage of intelligent agents, but problems concerning trust for automated interactions still represent a major obstacle for the adoption for agent-mediated e-commerce.

In the literature for trust-aware multi-agent networks, trust is defined as "a particular level of belief of an agent that the other agent will act or intend to act beneficially" [1]. Thus, trust implies a long-term future vision based on past reputation and previous performance.

The management of trust in distributed environments is widely studied among researchers. At present, the most widely used trust management model in distributed environments is the reputation-based model built on knowledge sharing network. Reputation is "the expectation about an agent's behavior based on observations of its 
past behavior" [1]. Generally, a reputation system receives, aggregates, and provides ratings about participants' past behavior. The ratings help participants decide whom to trust, encourage trustworthy behavior and deter dishonest people from participation [2]. eBay [3] and taboo [4] are successful examples of a reputation system. In $\mathrm{eBay}$, partners are rated by each other after the completion of a transaction. A central registry which stores these global ratings for each agent is openly accessible. This simple approach has its weakness when applied to unsupervised automated e-commerce environments. It is easily attacked by dishonest agents via inserting arbitrary number of fake ratings into the central registry. In multi-agent networks, only the individual trustworthiness judgment of one agent for another can fulfill the demand for security [9].

Other decentralized models based on knowledge sharing network [5, 6] are proposed for trust management in distributed systems. In these models, agents query about the trustworthiness of peers to interact with by sending broadcasts to collect advices from neighboring peers, regardless of the credibility of these peers. This method is not just inefficient, but also gives continued opportunities for dishonest peer agents to damage and influence the reputation network, because it does not consider the credibility of the knowledge sharing peers.

Through literature review, we have investigated the shortcomings of existing trust management models when applied to the autonomous agent-mediated ecommerce. Then, in the rest of the paper, we will extend the existing models which use knowledge sharing network to better incorporate both internal and external knowledge sources. The trust inference is based on the individual settings of each agent. Credibility of knowledge sharing peers is considered. Knowledge of trust is developed over time through learning from prior business interactions and problems related to peer agents' dishonesty can be solved. A book buying example is chosen to demonstrate the application of our model and verify the model's effectiveness. Finally, we close this paper with some conclusions and future work about the model.

\section{Proposed trust management model}

\subsection{The knowledge sharing network and the model elements}

In this section, we introduce our model for the management of trust between autonomous agents in agent-mediated e-commerce environments. This model is based on a knowledge sharing network (see Fig 1).

In one transaction, the Requesting Agent, who initiates the transaction, first locates the Target Agent that meets his requirements or expectations. Before the business interaction with the Target Agent, the Requesting Agent retrieves trust knowledge about the Target Agent from its individual repository which stores the knowledge extracted from past interactions. In addition, the Requesting Agent broadcasts a TrustRequest to all his known neighboring agents. Peer agents which have had interaction with the Target Agent may answer this TrustRequest with a TrustResponse and act as Recommending Agents. Then, the trust inference based on the diverse information sources can be carried out by the Requesting Agent. 
For each peer agent with whom the Requesting Agent directly interacted, it keeps two attributes in its Trusted agents Table TT: the target agent's id Tid and its own perception about the trustworthiness of target agent $T V_{I}$. To prevent dishonest Recommending Agents from providing false or poor recommendations, we introduce the concept of Recommending Credibility (RC). For each Recommending Agent who has provided recommendations about other peers to the Requesting Agent, it keeps a record in its Recommenders Table RT, including the Recommender's id Rid and the Recommending Credibility $R C$.

The trust value $\mathrm{TV}_{\mathrm{I}}$ and Recommending Credibility $\mathrm{RC}$ can be adjusted through interaction reviews. Interaction review is the key mechanism in our model to prevent dishonest recommendations. Also, knowledge of trust is learnt from interaction reviews and developed gradually over time.

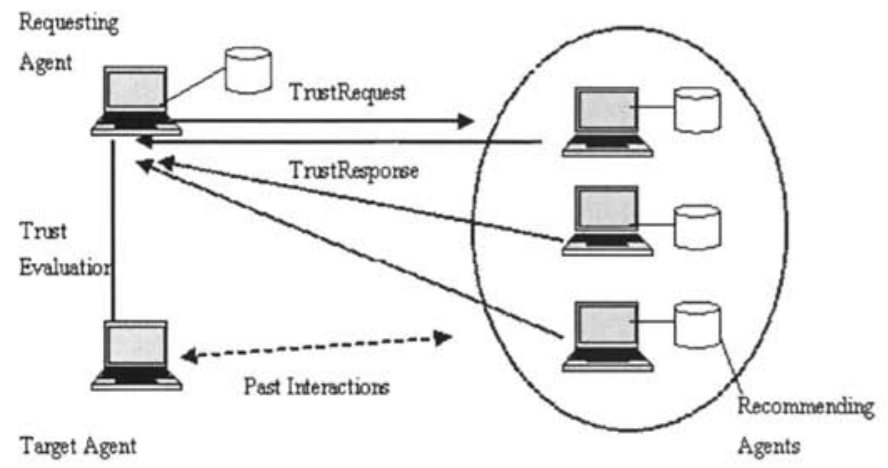

Fig. 5. Knowledge sharing network

\subsection{Trust inference}

After messages from several Recommending Agents being received, the Requesting Agent needs to pre-process the provided information. Different agent may define different trust value range, so the Requesting Agent have to scale the received trust values to its individual trustworthiness range using the supplied range $\left[\mathrm{t}_{\min }, \mathrm{t}_{\max }\right]$ in the TrustResponse. If the Requesting Agent's trust value range is $[0,5]$, the definition of each level see Table 1 . Then the received trust value $t_{v}$ from the Recommending Agent can be transformed to this range using Eq.(1).

$$
\mathrm{RTV}=\left(\mathrm{t}_{\mathrm{v}}-\mathrm{t}_{\min }\right) * 5 /\left(\mathrm{t}_{\max }-\mathrm{t}_{\min }\right) \quad \text { Eq. (1) }
$$

The Requesting Agent then retrieves Recommending Credibility (RC) of Recommending agents from its Recommenders Table (RT). If no credibility record exists, the Requesting Agent assigns an initial credibility value for the Recommending Agent to overcome the so called "newcomer problem" [7].

Subsequently, the TV and RC of Recommending Agents are fed into an inference engine. The summarized trust output that reflects the experience of all Recommending Agents $\mathrm{TV}_{\mathrm{E}}$ can be calculated using Eq.(2), where $\mathrm{n}$ represents the total number of all Recommending Agents who responded to the TrustRequest. 


$$
\mathrm{TV}_{\mathrm{E}}=\sum_{\mathrm{i}=1}^{\mathrm{n} T \mathrm{TV}_{\mathrm{i}}} \times \mathrm{RC}_{\mathrm{i}} / \sum_{\mathrm{i}=1}^{\mathrm{n}} \mathrm{RC}_{\mathrm{i}} \quad \text { Eq.(2) }
$$

In addition, the Requesting Agent looks up its own trust record about the Target Agent in past interactions from its Trusted agents Table TT. The internal query result $T V_{1}$ and the external summarized result $T V_{E}$ are incorporated to retrieve the overall trust value OTV for the Target Agent. Different Requesting Agent may weigh the internal direct trust and external reputation differently on different context. In order to provide this flexibility we can multiply an internal weight $\mathrm{W}_{\mathrm{I}}$ to the internal query result $\mathrm{TV}_{\mathrm{I}}$, and multiply an external weight $\mathrm{W}_{E}$ to the external summarized output $T V_{E}$. St: $W_{I}+W_{E}=1$. The overall trust value OTV can be calculated using Eq.(3).

$$
\mathrm{OTV}=\mathrm{TV}_{\mathrm{E}} \times \mathrm{W}_{\mathrm{E}}+\mathrm{TV}_{\mathrm{I}} \times \mathrm{W}_{\mathrm{I}} \quad \text { Eq.(3) }
$$

If the Target Agent is a new partner and there is no record about it in the trusted agents Table TT, the Requesting Agent assigns an initial trust value for the new Target Agent to overcome the so called "newcomer problem" [7].

Having inferred the final trustworthiness value for the Target Agent, now, the Requesting Agent can make decisions on its actions according to the interaction policies defined for each of the trust levels, and thus the Requesting Agent can now complete the business interaction.

Table 1. Trust levels

\begin{tabular}{|c|l|l|}
\hline Trust level & Linguistic definition & Trust value \\
\hline 0 & No trustworthy & $\mathrm{X}=0$ \\
\hline 1 & Very untrustworthy & $\mathrm{X} \in[0,1]$ \\
\hline 2 & Untrustworthy & $\mathrm{X} \in(1,2.5]$ \\
\hline 3 & Medium trustworthy & $\mathrm{X} \in(2.5,3.5]$ \\
\hline 4 & Trustworthy & $\mathrm{X} \in(3.5,4.5]$ \\
\hline 5 & Very trustworthy & $\mathrm{X} \in(4.5,5]$ \\
\hline
\end{tabular}

\subsection{Business interaction review}

After the interaction with the Target Agent is completed, an interaction review is carried out by the Target Agent to evaluate the Target Agent's actual performance by measuring the degree of fulfillment of the contract criteria which he negotiated with the Target Agent prior to their business interaction.

We make use of the modified Commitment methodology [8] for the performance evaluation. The evaluation of the overall performance is achieved by assessing the following two factors:

1. The commitment to each criterion of the contract $C_{k}$, range $[0,5]$

2. The importance of each criterion $I_{k}$, range $[0,5]$

The overall actual performance of the Target Agent in the interaction ITV (Instance Trust Value) can be expressed with the following expression where $\mathrm{m}$ represents the number of all criteria:

$$
\text { ITV }=\sum_{k=1}{ }^{\prime \prime} C_{k} \times I_{k} / \sum_{k=1} " I_{k}
$$

Then, the ITV, which represents the actual performance, can be used to adjust the trust value of the Target Agent and to measure the accuracy of recommendations provided by Recommending Agents, and then the Recommending Credibility of 
Recommending Agents can be adjusted. Frequent interaction reviews may bring overhead to agents, a review can be taken after every nth interaction.

\subsection{Trust and credibility adjustment}

After business review, the trust value of the Target Agent can be updated with the ITV value in the Trusted agents Table TT.

The adjustment of the Recommending Credibility value is achieved by measuring the accuracy of the given opinions in prior business interaction. The accuracy of a recommendation $\mathrm{AC}[0,1]$ can be calculated using Eq (5):

$$
\mathrm{AC}=1-|\mathrm{RTV}-\mathrm{ITV}| / 5 \quad \mathrm{Eq}(5)
$$

The recommendation is more accurate, $\mathrm{AC}$ value is more close to 1 . The Requesting Agent reinforces the $\mathrm{RC}$ value for opinions close to the actual performance and penalizes the $\mathrm{RC}$ value for opinions differing from the actual performance. A tolerance value $\varepsilon$ is introduced to determine whether the RC value needs to be increased or decreased. If $1-A C<\varepsilon$, then the $R C$ value should be increased, otherwise the $\mathrm{RC}$ value should be decreased.

Additionally, it is necessary to construct separate functions for the tasks of trust credibility reducing and increasing to simulate human behavior where trust is difficult to build and easy to lose [10]. Firstly, we make the following definitions: $\mathrm{RC}_{\max }=5, \mathrm{RC}_{\mathrm{avg}}=\sum_{\mathrm{i}=1}{ }^{\mathrm{n}} \mathrm{RC} \mathrm{i} / \mathrm{n}$, and $\mathrm{n}$ is the number of the Recommending Agents.

For the task of credibility increasing, a bell-shaped function can be used, it increases the agent's credibility value slowly if the existing credibility is relatively low or high but increases of the credibility value strongly if the existing credibility is medium[9]. The recommendation is more accurate, the increase is more significant. We use the following function for credibility increasing, see $\mathrm{Eq} \mathrm{(6).}$

$$
\Delta \mathrm{RC}^{+}=\mathrm{RC}_{\text {new }}-\mathrm{RC}=\mathrm{AC} \times\left(1-\mathrm{RC} / \mathrm{RC}_{\max }\right) \times \mathrm{e}^{-(\mathrm{RC}-\mathrm{RC}}{ }_{\text {avg }}{ }^{2} \mathrm{Eq}(6)
$$

$0<\mathrm{e}^{-(\mathrm{RC}-\mathrm{RC}}{ }_{\text {avg }}{ }^{2} \leq 1$, it is a bell-shaped function and reaches its maximum value when $\mathrm{RC}$ is close to the average credibility value.

$0 \leq \mathrm{AC} \leq 1, \mathrm{AC} \uparrow, \Delta \mathrm{RC}^{+} \uparrow$

$\Delta \mathrm{RC}^{+}=\mathrm{AC} \times \quad\left(1-\mathrm{RC} / \mathrm{RC}_{\max }\right) \times \mathrm{e}^{-\left(\mathrm{RC}-\mathrm{RC}_{\mathrm{avg}}{ }^{12} \leq 1-\mathrm{RC} / \mathrm{RC}_{\max } \quad<\mathrm{RC}_{\max }-\mathrm{RC},\right.}$, $\mathrm{RC}_{\text {new }}=\mathrm{RC}+\Delta \mathrm{RC}^{+}<\mathrm{RC}_{\text {max }}$.

So the credibility increasing function is reasonable and can fulfill the above requirements for credibility increasing.

For the task of credibility reducing, an exponential function can used, it reduces the agent's credibility slowly if the existing value is already at low and medium levels but decreases the credibility strongly if the existing credibility is high [9]. The recommendation is more inaccurate, the decrease is more significant. In our model, we use the following function for credibility reducing:

$$
\triangle R C^{=}=R C-R C_{n e w}=R C^{2} / R C_{\max } \times(1-A C) \times \mathrm{e}^{(R C-R C}{ }_{\max }^{\prime} \quad \text { Eq (7) }
$$
$\mathrm{RC}_{\max }$

$R C \leq R C_{\max }$, so $0<\mathrm{e}^{(R C-R C}{ }_{\max }{ }^{3} \leq \mathrm{e}^{0}=1$, it decreases sharply when $R C$ is close to

$$
\begin{aligned}
& 0 \leq \mathrm{AC} \leq 1,0 \leq(1-\mathrm{AC}) \leq 1, \mathrm{AC} \uparrow,(1-\mathrm{AC}) \downarrow, \Delta \mathrm{RC}^{-} \downarrow \\
& \left.\Delta \mathrm{RC}^{-}=\mathrm{RC}^{2} / \mathrm{RC}_{\max } \times(1-\mathrm{AC}) \times \mathrm{e}^{(\mathrm{RC}-\mathrm{RC}}{ }_{\max }\right)^{\prime} \leq \mathrm{RC}^{2} / \mathrm{RC}_{\max }<\mathrm{RC}, \mathrm{RC}_{\text {new }}=\mathrm{RC}-\Delta \mathrm{RC}>0 .
\end{aligned}
$$

So the credibility decreasing function is reasonable and can fulfill the above requirements for credibility decreasing. 
The carefully defined functions for Recommending Credibility adjustment can prevent possible periodic dishonesty behavior of Recommending Agents [2].

\section{Application example}

To demonstrate the application of our model and verify its effectiveness, we choose the simple book buying example as the business scenario. The agent owner specifies his requirements for the book buying as in Table 2.

Table 2. The book buying example

\begin{tabular}{|c|c|c|}
\hline Item & Requirement & Importance \\
\hline Topic & Knowledge management in e-commerce & 4.5 \\
\hline Price & $<=\$ 25$ & 3 \\
\hline Delivery & Within 5 days & 3.5 \\
\hline
\end{tabular}

Before the agent can work, the agent owner needs to define a set of variables and policies reflecting his individual security requirements. For example, he needs to specify the tolerance value to determine whether the $\mathrm{RC}$ value needs to be increased or decreased. Additionally, the agent owner specifies weights used for the incorporation of internal and external knowledge. In our example, the settings are as following:

Tolerance value: $\varepsilon=8 \%$

Weights for internal and external knowledge: $\mathrm{W}_{\Gamma}=0.45, \mathrm{~W}_{\mathrm{E}}=0.55$

The internal trust value is 4.2 . The Requesting Agent received 4 TrustResponses from 4 Recommending Agents (see Table 3). RC values of the 4 Recommending Agents are listed aside.

Table 3. TrustResponses and $R C$ values

\begin{tabular}{|c|c|l|}
\hline Recommending Agent & Recommended Trust value and range & RC value \\
\hline $\mathrm{A} 1$ & $6.5,[0,10]$ & 4.5 \\
\hline $\mathrm{A} 2$ & $4.5,[0,10]$ & 4.0 \\
\hline $\mathrm{A} 3$ & $0.8,[0,1]$ & 2.8 \\
\hline $\mathrm{A} 4$ & $3.6,[0,5]$ & 3.5 \\
\hline
\end{tabular}

Using the previously defined user settings, the Requesting Agent can immediately start its trust inference.

Step 1 Information pre-processing

Transform the received trust values to range [0,5] using Eq.(1):

RTV1 $=3.25 \quad$ RTV2 $=2.25 \quad$ RTV3 $=4.0$ RTV4 $=3.6$

Step 2 Trust inference

The overall trust value OTV can be inferred using Eq.(2) and Eq.(3):

$\mathrm{OTV}=(3.25 * 4.5+2.25 * 4.0+4.0 * 2.8+3.6 * 3.5) * 0.55 /(4.5+4.0+2.8+3.5)+4.2 * 0.45$ 
$=3.65$

The trust level is 4: 'Trustworthy' which allows the Requesting Agent to interact with the Target Agent.

Step 3 Business interaction review

After the business interaction, the Requesting Agent reviews the performance of the delivered goods and services. Fulfillment of each criterion is evaluated by the Requesting Agent as in Table 4.

Table 4. Commitment of each criterion

\begin{tabular}{|l|c|l|}
\hline Item & Commitment & Importance \\
\hline Topic & 3.5 & 4.5 \\
\hline Price & 5 & 3 \\
\hline Delivery & 2.5 & 3.5 \\
\hline
\end{tabular}

The overall actual performance of the Target Agent in this interaction ITV (Instance Trust Value) can be calculated using Eq.(4):

$\mathrm{ITV}=(3.5 * 4.5+5 * 3+2.5 * 3.5) /(4.5+3+3.5)=3.59$

Step 4 Trust and credibility adjustment

1) Update the internal trust value:

$\mathrm{TV}_{1}=\mathrm{ITV}=3.59$

2) Determine whether the credibility of each Recommending Agent should be increased or decreased (Tolerance value $\varepsilon=8 \%$ ):

$\mathrm{A} 1: \mathrm{AC} 1=1-[3.25-3.59 \mid / 5=0.932,1-\mathrm{AC} 1=0.068<\varepsilon \quad$ increase credibility

$\mathrm{A} 2: \mathrm{AC} 2=1-\mid 2.25-3.59 / / 5=0.732,1-\mathrm{AC} 2=0.268>\varepsilon$ decrease credibility

$\mathrm{A} 3: \mathrm{AC} 3=1-|4.0-3.59| / 5=0.918,1-\mathrm{AC} 3=0.082>\varepsilon \quad$ decrease credibility

$\mathrm{A} 4: \mathrm{AC} 4=1-|3.6-3.59| / 5=0.998,1-\mathrm{AC} 4=0.002<\varepsilon \quad$ increase credibility

3) Adjust credibility values:

$\mathrm{RC}_{\text {avg }}=(4.5+4.0+2.8+3.5) / 4=3.7$

$\mathrm{RCl}_{\text {new }}=4.5+0.932 *(1-4.5 / 5) * \mathrm{e}^{-(4.5-3.7) 2}=4.5+0.049=4.549$

$\mathrm{RC2} 2_{\text {new }}=4.0-4.0 * 4.0 / 5.0^{*} 0.268 * \mathrm{e}^{(4.0-5.0)}=4.0-0.315=3.685$

$\mathrm{RC} 3_{\text {new }}=2.8-2.8 * 2.8 / 5.0 * 0.082 * \mathrm{e}^{(2.8-5.0)}=2.8-0.005=2.795$

$R C 4_{\text {new }}=3.6+0.998 *(1-3.5 / 5.0)^{*} \mathrm{e}^{-(3.5-3.7) 2}=3.6+0.288=3.888$

The results show that honest and accurate recommendations are rewarded slightly; but poor or fake recommendations are punished significantly when the RC value is already high. This strategy can prevent dishonest recommenders from cheating periodically.

\section{Conclusion and future work}

Following our knowledge sharing network based model, agents can reach a comprehensive decision about the trust of their business partners. By collaborating in a trust network, agents can benefit from the external knowledge shared by other peer agents. The external knowledge can be incorporated with their internal knowledge to 
infer trust values for various potential business partners to facilitate the selection of the best matching and trustworthy business partner. The trust inference is based on the individual settings of each agent, so it can fulfill the demand for security. Furthermore, we introduced a mechanism for preventing dishonest recommendations through Recommending Credibility adjustment based on the outcome of interaction review. Using the proposed model the agents can build up a growing trust knowledge base through learning from prior interactions.

Due to the subjectivity and uncertainty contained in the individual notions and definitions of trust, trust management demands a flexible and adjustable model. In the future work, we plan to study fuzzy inference in our trust management model.

\section{References}

1. S.Y. Stephen, Managing Trust in Distributed Agent Systems, ATC 2006, LNCS 4158, Springer-Verlag Berlin Heidelberg, 17-25(2006).

2. A.A. Farag, Trust Modeling and Its Applications for Peer-to-Peer Based Systems, PhD Thesis, Computer Science, University of Mantoba(2004).

3. eBay homepage. http://www.ebay.com.

4. taobao homepage. http://www.taobao.com.

5 E. Damiani, D. Vimercati, S. Paraboschi, P. Samarati and F. Violante, A reputation-based approach for choosing reliable resources in peer-to-peer networks, Proceedings of the 9th ACM Conference on Computer and Communications Security, 207-216(2002).

6 L.Xiong and L. Liu, A Reputation-Based Trust Model for peer-to-peer ecommerce Communities, IEEE International conference on E-Commerce (CEC'03), 270-280(2003).

7. S. Ruohomaa, Lea Kutvonen Trust Management Survey, Trust Management: Second International Conference, 77-92(2005).

8. F.K. Hussain, E. Chang and T.S. Dillon, Trustworthiness and CCCI metrics for assigning trustworthiness in P2P communication, Intl. J. Comput. Syst, 19 (4), 95-112(2004)

9. S. Stefan et al, Applying a Fuzzy Trust Model to E-Commerce Systems, AI 2005, LNAI 3809, Springer-Verlag Berlin Heidelberg, 318 - 329(2005).

10.M A. Patton and A. Josang, Technologies for Trust in Electronic Commerce, Electronic Commerce Research ,4, 9-21(2004). 